Hybrid cochlear implantation : quality of life, quality of hearing, and working performance compared to patients with conventional unilateral or bilateral cochlear implantation

\author{
Harkonen, Kati
}

$2017-10$

Harkonen , K , Kivekas , I, Kotti , V , Sivonen, V \& Vasama, J-P 2017 , ' Hybrid cochlear implantation : quality of life, quality of hearing, and working performance compared to patients with conventional unilateral or bilateral cochlear implantation ', European Archives of Oto-Rhino-Laryngology , vol. 274 , no. 10 , pp. 3599-3604 . https://doi.org/10.1007/s00405-017-4690-9

http://hdl.handle.net/10138/245626

https://doi.org/10.1007/s00405-017-4690-9

publishedVersion

Downloaded from Helda, University of Helsinki institutional repository.

This is an electronic reprint of the original article.

This reprint may differ from the original in pagination and typographic detail.

Please cite the original version. 


\title{
Hybrid cochlear implantation: quality of life, quality of hearing, and working performance compared to patients with conventional unilateral or bilateral cochlear implantation
}

\author{
Kati Härkönen ${ }^{1,2}$ (1) Ilkka Kivekäs ${ }^{1,2} \cdot$ Voitto Kotti $^{2} \cdot$ Ville Sivonen $^{3} \cdot$ \\ Juha-Pekka Vasama ${ }^{2}$
}

Received: 23 February 2017/ Accepted: 25 July 2017/Published online: 31 July 2017

(C) Springer-Verlag GmbH Germany 2017

\begin{abstract}
The objective of the present study is to evaluate the effect of hybrid cochlear implantation (hCI) on quality of life $(\mathrm{QoL})$, quality of hearing $(\mathrm{QoH})$, and working performance in adult patients, and to compare the long-term results of patients with hCI to those of patients with conventional unilateral cochlear implantation $(\mathrm{CI})$, bilateral CI, and single-sided deafness (SSD) with CI. Sound localization accuracy and speech-in-noise test were also compared between these groups. Eight patients with highfrequency sensorineural hearing loss of unknown etiology were selected in the study. Patients with hCI had better long-term speech perception in noise than uni- or bilateral CI patients, but the difference was not statistically significant. The sound localization accuracy was equal in the hCI, bilateral CI, and SSD patients. QoH was statistically significantly better in bilateral CI patients than in the others. In hCI patients, residual hearing was preserved in all patients after the surgery. During the 3.6-year follow-up, the mean hearing threshold at $125-500 \mathrm{~Hz}$ decreased on average by $15 \mathrm{~dB} \mathrm{HL}$ in the implanted ear. QoL and working performance improved significantly in all CI patients. Hearing outcomes with hCI are comparable to the results of bilateral CI or CI with SSD, but hearing in noise and sound localization are statistically significantly better than with unilateral CI. Interestingly, the impact of CI on
\end{abstract}

Kati Härkönen

kati.a.harkonen@gmail.com

1 Department of Otorhinolaryngology, University of Tampere, Tampere, Finland

2 Department of Ear and Oral Diseases, Tampere University Hospital, P.O. Box 2000, 33521 Tampere, Finland

3 Department of Otorhinolaryngology, Helsinki University Central Hospital, Helsinki, Finland
QoL, QoH, and working performance was similar in all groups.

Keywords Hybrid cochlear implant - Quality of life · Working ability · Quality of hearing · Residual hearing

\section{Introduction}

Cochlear implant (CI) rehabilitation can be categorized into four different types depending on the implanted condition; unilateral, bilateral, bimodal (CI and contralateral hearing aid), and hybrid. Electroacoustic stimulation with a hybrid cochlear implant (hCI) is an option for patients with severe, high-frequency sensorineural hearing impairment.

Cochlear implantation, either uni- or bilateral, has a positive effect on quality of life (QoL) and quality of hearing $(\mathrm{QoH})$ [1]. Despite constantly developing processor techniques, the QoH with traditional CI may remain relatively unnatural and therefore hearing in noise is often challenging $[1,2]$. Interestingly, hCIs have been shown to improve hearing in noise and even music perception over traditional CIs [3-5]. Moreover, advanced surgical techniques and less traumatic electrodes have led to better preservation of cochlear structures, providing a better basis for good speech perception with preserved residual hearing.

Sound localization with a single CI (with or without a contralateral hearing aid) is poor. Bilateral CI use improves sound localization, but it is still much poorer than in normal hearing controls [1]. Because of their relatively good low-frequency residual hearing, hCI patients are expected to have better sound localization than patients with conventional CI. Interestingly, Moteki et al. have shown that bilateral hCIs effectively improved speech perception in 
noise and sound localization in patients with high-frequency hearing loss [6]. Gifford et al. and Adunka et al. have shown that, in hCI patients, performance in complex hearing environments-for example, in restaurant noiseis better with electro-acoustic stimulation than with electric stimulation alone [3, 7]. Rader et al. found that bimodal hCIs (with a hearing aid in the contralateral ear) significantly improved speech perception in noise compared to bilateral cochlear implantation [8]. In most workplaces, the hearing environment is complex, and hearing loss may cause difficulties in working performance. Improved hearing in noise and more accurate sound localization should lead to better performance at work and in everyday life. These benefits have not been evaluated in hCI patients.

The purpose of this study was twofold: (1) to evaluate the long-term effects of hCIs on QoL, QoH, and working performance in adult patients; and (2) to compare these results to patients with conventional unilateral or bilateral CI.

\section{Materials and methods}

We invited all eight hCI patients operated in our hospital to participate in the study. All patients (5 women and 3 men) with a mean implantation age of 49 years (range 25-70 years) responded and were included. Six patients had unilateral hCIs and two patients had bilateral hCIs. Five patients with unilateral hCIs used a hearing aid (HA) in the contralateral ear. Six patients used acoustic amplification in the implanted ear (i.e., electro-acoustic stimulation) and two patients used only the electric stimulation mode. Bilateral high-frequency sensorineural hearing loss etiology was unknown in all patients. The duration between the hCI implantation and the study was, on average, 3.6 years (range 1.7-5.1 years). The study was conducted at Tampere University Hospital, Tampere, Finland, and approved by the Ethics Committee of Pirkanmaa Hospital District, Tampere University Hospital. Detailed patient data are shown in Table 1.

Comparison groups were single-sided deafness (SSD), patients with unilateral CI, and sequentially bilaterally implanted patients. The SSD group included seven patients (five women and two men, mean age 48 years, range 36-61 years) and the etiology was sudden deafness of unknown origin in five patients and stapes surgery in two patients. The average time between the deafness and cochlear implantation was 2.5 years (range $1-7$ years). All patients had normal contralateral hearing. The bilateral CI group consisted of 15 patients ( 9 females, 6 males, mean age 41 years, range 19-58 years). The etiology was bilateral progressive postlingual sensorineural hearing loss in 14 patients, and 1 patient had severe bilateral congenital hearing impairment. Ten patients had used an HA in the contralateral ear before the second CI surgery. The first CI had been implanted, on average, 4.7 years (range 1-14 years) before the second CI. The follow-up period after CI surgery was 1 year in both comparison groups. Detailed information about these control groups can be found in our previous studies [1, 2].

The Cochlear ${ }^{\mathrm{TM}}$ Nucleus ${ }^{\circledR}$ Hybrid L24 Cochlear Implant System provides electrical stimulation to the basal section of the cochlea while protecting the apical section to provide benefits from acoustic stimulation. This combination of stimulation is indicated in patients with mild to moderate hearing loss in the low frequencies and severe to profound hearing loss in the high frequencies. The implant's active array is $15 \mathrm{~mm}$ in length with 22 half-band electrode contacts inserted through the round window or cochleostomy into the cochlea. The typical insertion depth is $16 \mathrm{~mm}$, indicated by a stopper to control the insertion depth. The electrode diameter is $0.4 \mathrm{~mm}$ at the basal end and $0.25 \mathrm{~mm}$ at the apical end. In this study, cochlear implantations were performed with a standard mastoidectomy and the facial recess approach. The round window niche was identified and the bony overhang was carefully removed to provide good access to the round window membrane. The electrode insertion was performed via an incision to the round window membrane.

Preoperative hearing and speech discrimination (SD) were measured with recorded bisyllabic, phonetically balanced words in the Finnish language that have been validated for adults [9]. Residual hearing and SD were measured 1 month after the surgery and at the end of the follow-up. Binaural hearing and hearing with an hCI alone were tested in a sound field. Hearing in the non-implanted ear with and without HA was tested after the follow-up.

The patients' QoL, QoH, and working performance were evaluated using specific questionnaires, which were completed after the follow-up. The patients' QoL was measured by the Glasgow Benefit Inventory (GBI) questionnaire [10]. The GBI scores after the implantation of an hCI depict the positive or negative impact of hCIs on the patient's QoL compared to the former condition. The test contains 18 questions and the response to each question is placed on a five-point scale ranging from a large deterioration to a large improvement in health status. The GBI consists of a total score and three subscores (general, social support, and physical health). The total score is transposed onto a benefit scale ranging from -100 (maximal negative benefit) through 0 (no benefit) to +100 (maximal positive benefit).

QoH was measured with the Speech, Spatial and Qualities of Hearing Scale (SSQ; version 3.1.2) questionnaire, where patients use a visual analog scale (VAS; from 0 to 10) to evaluate their current hearing. The SSQ 
Table 1 Patient demographics

\begin{tabular}{llllllll}
\hline No & F/M & Age (years) & hCI (ear) & HA (other ear) & Years implanted & Processor & Acoustic stimulation \\
\hline 1 & M & 25 & Right & + & 5 & CP910 & + \\
2 & M & 46 & Both & & 5 and 4 & CP810 + CP810 & - \\
3 & M & 70 & Right & - & 3 & CP910 & + \\
4 & F & 45 & Right & + & 3 & CP910 & + \\
5 & F & 51 & Right & + & 3 & CP810 & - \\
6 & F & 62 & Left & + & 2 & CP910 & + \\
7 & F & 33 & Both & & 5 and 4 & CP910 + freedom & + \\
8 & F & 57 & Right & + & 3 & CP910 & + \\
\hline
\end{tabular}

$F$ female, $M$ male, $h C I$ hybrid cochlear implant, $H A$ hearing aid

questionnaire is divided into three categories: speech intelligibility, spatial perception, and sound quality. The questionnaire measuring working performance has not been validated. One patient was retired and did not fill this questionnaire.

To assess the effect of electro-acoustic stimulation on hearing, the patients' speech recognition in noise and localization abilities were measured via five loudspeakers placed at $0^{\circ} \pm 45^{\circ}$ and $\pm 90^{\circ}$ of azimuth in the horizontal plane in a sound-field test room. Speech-in-noise and localization tests were carried out after the postoperative period following the implantation of the hCI.

In the speech-in-noise test, phonetically balanced bisyllabic Finnish words [9] were presented at a level of $65 \mathrm{~dB}$ SPL from the loudspeaker at $0^{\circ}$ of azimuth. The noise was an unmodulated artificial signal with a long-term spectrum corresponding to human speech [11]. The noise was uncorrelated and presented from the other four loudspeakers, and its level was varied in $5 \mathrm{~dB}$ steps to achieve speech recognition scores as a function of signal-to-noise ratio (SNR). The six SNRs in the present study were -10 , $-5,0,+5,+10$, and $+15 \mathrm{~dB}$. In the localization test, short speech segments were presented randomly from each of the five loudspeakers. The presentation level was $65 \mathrm{~dB}$ SPL and it was roved within $\pm 5 \mathrm{~dB}$ to avoid the participants using loudness as a cue to localize sound. In the analysis, sound localization accuracy was quantified by an error index (EI) ranging from 0 to 1 , with 0 corresponding to perfect localization accuracy and 1 being chance performance.

In the assessment, the participants were instructed to face the frontal loudspeaker at $0^{\circ}$ of azimuth and to not move their heads during the test. In the speech-in-noise test, they were instructed to repeat the word they heard, and in the localization test, to name the loudspeaker they thought the sound was emanating from. Similar data had earlier been collected for sequentially bilaterally implanted adults, as well as for a normally hearing control group [1] and SSD patients implanted unilaterally with a cochlear implant [2]. Although background and etiological factors play a major role in cochlear implant outcomes, the aim of the present assessment was to arrive at a basic understanding of how well the recipients of hCIs fared in comparison to the other two groups of cochlear implantees. More details on the test setup are found in Härkönen et al. $[1,2]$.

The data were analyzed with SPSS for Windows statistical software, version 19.0. The comparison between the pre- and postoperative health (GBI) was performed using a nonparametric test (the Wilcoxon signed-rank test). The Mann-Whitney $U$ test and the Kruskal-Wallis test were used when comparing the hCI, SSD, and unilateral and bilateral CI groups. The differences were considered statistically significant at a $p$ value $<0.05$.

\section{Results}

The GBI scores showed a positive effect for $\mathrm{CI}$ on $\mathrm{QoL}$ in all groups. In hCI patients, the mean total GBI score was +44 at the end of the follow-up $(p=0.012)$. The mean subscore was $+68(p=0.011)$ for general health, +2 for social support (not significant; ns), and -10 for physical health (ns). One patient was diagnosed with breast cancer during the study and this probably influenced her physical health subscore. In comparison to the other CI groups, the mean total GBI score was statistically significantly higher for the hCI patients than for the SSD patients $(p=0.012)$ (see Table 2).

Working performance clearly improved after the hCI (see Table 3). The patients managed much better at work and the implantation had a positive influence on their career planning. Communication with co-workers and speaking on the phone were also much easier. The patients were more active in their working environment and more alert after the working day. Working performance 
Table 2 The mean long-term Glasgow Benefit Inventory scores

\begin{tabular}{llllc}
\hline & hCI & Unilat. CI & Bilat. CI & SSD + CI \\
\hline Total (SD) & $+44(9)$ & $+43(19)$ & $+39(17)$ & $+28(10)$ \\
General & $+68(12)$ & $+60(26)$ & $+56(27)$ & $+42(18)$ \\
Social support & $+2(19)$ & $+12(20)$ & $+6(12)$ & $+7(9)$ \\
Physical health & $-10(25)$ & $+8(19)$ & $+8(23)$ & $-5(13)$ \\
\hline
\end{tabular}

$C I$ cochlear implant, $h C I$ hybrid cochlear implant, $S S D$ single-sided deafness, $S D$ standard deviation

improved after cochlear implantation in all comparison groups $[1,2]$.

In the hCI patients, the SSQ score was 6.2 for sound quality, 5.4 for speech intelligibility, and 5.5 for spatial perception. In comparison to the other groups, speech intelligibility was statistically significantly better in bilateral CI patients than in hCI or SSD patients $(p=0.034)$ (see Table 4).

The mean EI score was 0.34 in the hCI patients. This result is in line with patients with bilateral CI (0.31) and SSD (0.31). The sound localization score was 0.73 in unilateral CI patients, which was statistically significantly worse than the other groups $(p<0.004)$.

Figure 1 demonstrates the speech perception in noise in normal hearing controls and all CI groups. SSD patients had statistically significantly better speech perception scores than the other CI groups $(p<0.027)$. The scores were clearly the worst in patients with a unilateral CI.

At the end of the follow-up, the mean decrease in hearing at $125 \mathrm{~Hz}$ was $11 \mathrm{~dB} \mathrm{HL}$ in the implanted ear and $2 \mathrm{~dB}$ HL in the non-operated ear. The decrease was 14 and $10 \mathrm{~dB} \mathrm{HL}$ at $250 \mathrm{~Hz}$ and 19 and $19 \mathrm{~dB} \mathrm{HL}$ at $500 \mathrm{~Hz}$, respectively. One patient was excluded from this study because she completely lost her residual hearing during chemotherapy (see Fig. 2).
Table 4 The mean long-term speech, spatial and qualities of hearing scale scores

\begin{tabular}{lcccc}
\hline & hCI & Unilat. CI & Bilat. CI & SSD + CI \\
\hline Sound quality (SD) & $6.2(1.5)$ & $6.7(1.3)$ & $7.6(1.1)$ & $7.0(1.1)$ \\
Speech intelligibility & $5.4(1.1)$ & $5.7(1.3)$ & $7.0(1.6)$ & $5.7(1.6)$ \\
Spatial perception & $5.5 .(1.5)$ & $3.0(1.5)$ & $6.3(1.4)$ & $5.1(1.4)$ \\
\hline
\end{tabular}

$h C I$ hybrid cochlear implant, $S S D$ single-sided deafness, $S D$ standard deviation

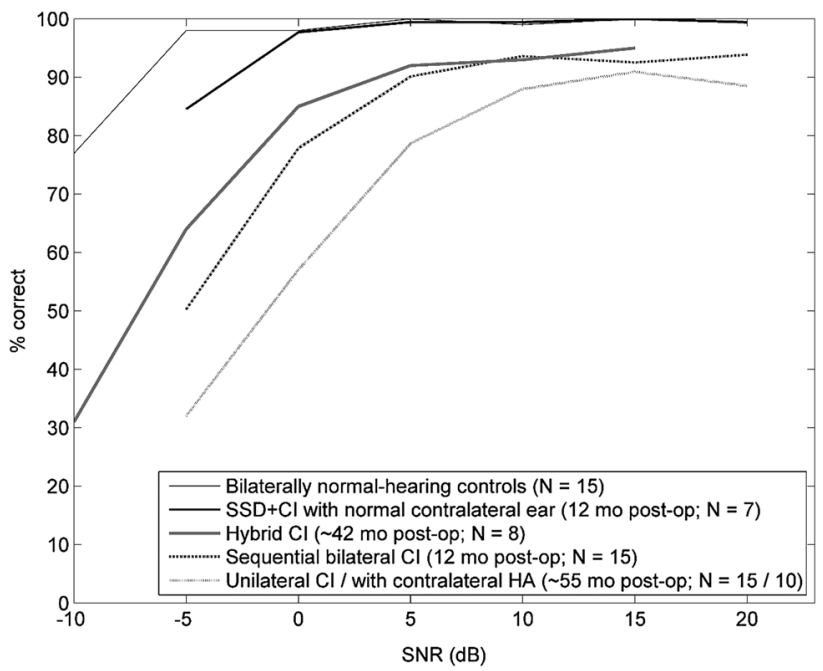

Fig. 1 The mean best-aided speech perception in noise. SNR signalto-noise ratio, $S S D$ single-sided deafness

After the follow-up, the mean best-aided sound-field PTA was $25 \mathrm{~dB}$ HL and the SD was $87 \%$. The mean PTA with an hCI alone was $27 \mathrm{~dB} \mathrm{HL}$ and the SD was $88 \%$. In the non-implanted ear, the best-aided PTA was $64 \mathrm{~dB} \mathrm{HL}$ and the SD was $56 \%$.
Table 3 Questionnaire for working performance with hCI
1. How much has the hCI helped you to do your work?
(a) Very much
(b) Moderately
(c) A little
(d) No change
(e) Worsened

2. How much has the hCI positively influenced your career development or planning?
(a) Very much
(b) Moderately
(c) A little
(d) No change
(e) Worsened

3. How much more active have you been in your working environment after the hCI?
(a) Very much
(b) MODERATELY
(c) A little
(d) No change
(e) Decreased activity
4. Has the hCI decreased your fatigue after the workday?
(a) Very much
(b) Moderately
(c) A little
(d) No change
(e) Increased fatigue

5. Is it easier to communicate with your co-workers after the hCI?
(a) Very much
(b) Moderately
(c) A little
(d) No change
(e) Worsened

6. Is it easier to speak on the phone after the hCI?
(a) Very much
(b) Moderately
(c) A little
(d) No change
(e) Worsened 


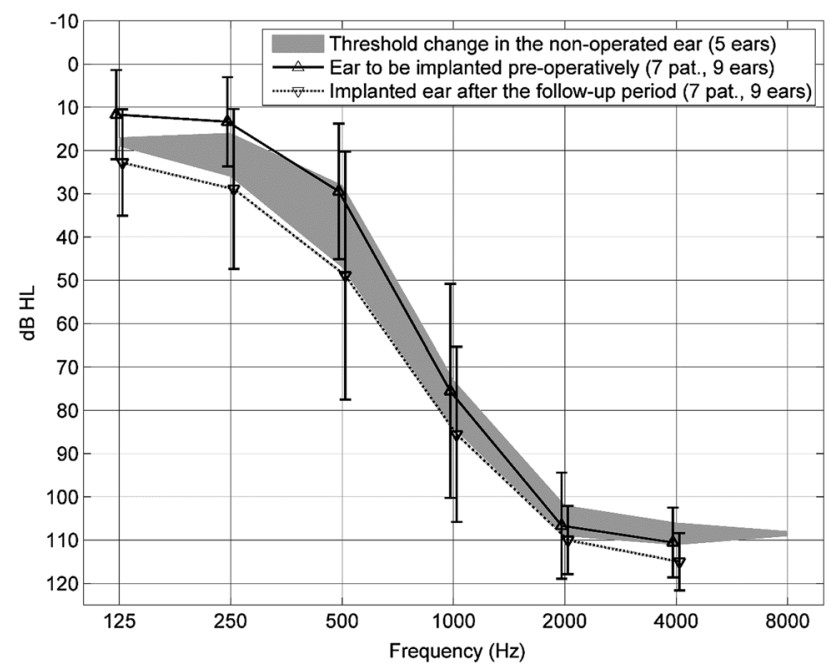

Fig. 2 The mean pre- and postoperative hearing thresholds and the change in hearing thresholds in the non-operated ear

\section{Discussion}

This study shows that patients with hybrid and conventional CIs experienced a positive impact from cochlear implantation on their well-being and working performance. QoL increased more in hCI patients than in SSD patients. The reason for this might be that, with one normally hearing ear, SSD patients managed quite well already before the cochlear implantation.

Rader et al. have shown that patients with an hCI and a contralateral HA had statistically significantly better speech perception in noise than patients with bilateral CIs, indicating that binaural interaction between the $\mathrm{hCI}$ in one ear and residual acoustic hearing in the opposite ear enhances speech perception in complex noise situations [8]. This is in agreement with our study, although our findings were not statistically significant. Gifford et al. and Adunka et al. found that the performance in noise of patients with hCIs was better with electro-acoustic stimulation than with electric stimulation alone [3, 7]. However, in these studies the comparison between electro-acoustic and electric stimulation was made within one ear by switching acoustic gain on/off. In our study, the comparison was performed between patients with hCIs and conventional CIs.

The mean long-term sound localization accuracy was similar in hCI, bilateral CI, and SSD patients. We have shown earlier that sound localization improved statistically significantly after sequential bilateral cochlear implantation and when the deaf ears of patients with SSD were implanted. The mean EI score decreased from 0.73 to 0.31 after the second CI and from 0.94 to 0.31 in the SSD patients $[1,2]$. Unfortunately, preoperative sound localization scores were not available for the hCI patients. However, in two patients with bilateral hCIs, the sound localization was tested before the second implant. One patient had normal localization accuracy before and after the surgery. The other patient's preoperative EI was 0.42 with a contralateral HA and 0.22 with two hCIs without acoustic stimulation. In severe uni- or bilateral hearing impairment, moderately good sound localization can only be achieved by cochlear implantation(s) that enhances binaural hearing.

There is always a risk with cochlear implantation that residual hearing will disappear partially or entirely. The new shorter electrode designs and soft surgery techniques aim at good long-term preservation of residual hearing. It is probable that the shorter the electrode, the less is the risk for hearing loss. Jurawitz et al. have shown that residual hearing was preserved for the majority of the 197 implanted patients with the Hybrid L24 and the CI422 implant [12]. Patients implanted with the Hybrid L24 implant demonstrated greater stability and less median hearing loss over time than those with the CI422 implant. Talbot et al. have shown that $13 \%$ of hCI patients had a total loss of residual low-frequency hearing after the implantation [5]. All our patients had good hearing preservation with the Hybrid L24 implant immediately after the surgery. During the 3.6-year follow-up, the mean hearing threshold at $125-500 \mathrm{~Hz}$ decreased on average by $15 \mathrm{~dB}$ HL in the implanted ear and $10 \mathrm{~dB}$ HL in the nonimplanted ear. At $500 \mathrm{~Hz}$, the mean decrease was $19 \mathrm{~dB}$ HL in both ears. Interestingly, Wiley et al. [13] have calculated the rate of change in hearing thresholds for 48- to 59-year-old healthy men to be approximately $0.4 \mathrm{~dB}$ per year at $500 \mathrm{~Hz}$. The more rapid hearing deterioration in our patients clearly exceeds Wiley's findings and probably results from the initial surgical trauma and/or unknown etiological factors.

This study confirms the clinical observations that cochlear implantation improves quality of life, quality of hearing, and working performance in patients with severe sensorineural high-frequency hearing loss, and the results are comparable with patients with single-sided deafness and those with bilateral implants. Furthermore, good speech perception and sound localization cannot be achieved with unilateral CI only. Due to the small number of patients and large variation, for example, in duration of deafness, the duration of $\mathrm{CI}$ experience, and cause of deafness among the patients, it is difficult to create matched pairs for comparison. Therefore, our results may reflect individual experience and performance, and outcomes may not be directly derived from the CI treatment option. To uncover these differences, larger multi-center studies are needed. 


\section{Compliance with ethical standards}

Conflict of interest The authors have no funding, financial relationships, or conflicts of interest to disclose.

Funding Dr. Kati Härkönen was supported by grants from the Finnish Cultural Foundation, the Finnish Otorhinolaryngology and Head and Neck Surgery Foundation, the Finnish Medical Foundation, and the Finnish Audiological Society.

Ethical approval All procedures performed in studies involving human participants were in accordance with the ethical standards of the institutional and/or national research committee and with the 1964 Helsinki Declaration and its later amendments or comparable ethical standards.

Informed consent Informed consent was obtained from all individual participants included in the study.

\section{References}

1. Härkönen K, Kivekäs I, Rautiainen M, Kotti V, Sivonen V, Vasama JP (2015) Sequential bilateral cochlear implantation improves working performance, quality of life, and quality of hearing. Acta Otolaryngol 135:440-446

2. Härkönen K, Kivekäs I, Rautiainen M, Kotti V, Sivonen V, Vasama JP (2015) Single-sided deafness: the effect of cochlear implantation on quality of life, quality of hearing, and working performance. ORL J Otorhinolaryngol Relat Spec 77:339-345

3. Gifford RH, Dorman MF, Skarzynski H, Lorens A, Polak M, Driscoll CLW et al (2013) Cochlear implantation with hearing preservation yields significant benefit for speech recognition in complex listening environments. Ear Hear 34:413-425
4. Gfeller KE, Olszewski C, Turner C, Gantz B, Oleson J (2006) Music perception with cochlear implants and residual hearing. Audiol Neurootol 11:12-15

5. Talbot KN, Hartley DEH (2008) Combined electro-acoustic stimulation: a beneficial union? Clin Otolaryngol 33:536-545

6. Moteki H, Kitoh R, Tsukada K, Iwasaki S, Nishio S, Usami S (2015) The advantages of sound localization and speech perception of bilateral electric acoustic stimulation. Acta Otolaryngol 135:147-153

7. Adunka OF, Dillon MT, Adunka MC, King ER, Pillsbury HC, Buchman CA (2013) Hearing preservation and speech perception outcomes with electric-acoustic stimulation after 12 months of listening experience. Laryngoscope 123:2509-2515

8. Rader T, Fastl H, Baumann U (2013) Speech perception with combined electric-acoustic stimulation and bilateral cochlear implants in a multisource noise field. Ear Hear 34:324-332

9. Jauhiainen T (1974) An experimental study of the auditory perception of isolated bi-syllable Finnish words. Dissertation, University of Helsinki, Finland

10. Robinson K, Gatehouse S, Browning GG (1996) Measuring patient benefit from otorhinolaryngological surgery and therapy. Ann Otol Rhinol Laryngol 105:415-422

11. Dreschler WA, Verschuure H, Ludvigsen C, Westermann S (2001) ICRA noises: artificial noise signals with speech-like spectral and temporal properties for hearing instrument assessment. International collegium for rehabilitative audiology. Audiology 40:148-157

12. Jurawitz M, Buchner A, Harpel $T$, Schussler M, Majdani $O$, Lesinski-Schiedat A, Lenarz T (2014) Hearing preservation outcomes with different cochlear implant electrodes: nucleus HybridTM-L24 and nucleus FreedomTM CI422. Audiol Neurootol 19:293-309

13. Wiley TL, Chappell R, Carmichael L, Nondahl DM, Cruickshanks KJ (2008) Changes in hearing thresholds over 10 years in older adults. J Am Acad Audiol 19:281-292 